\title{
Ferrimagnetic $120^{\circ}$ magnetic structure in $\mathrm{Cu}_{2} \mathrm{OSO}_{4}$
}

\author{
Virgile Yves Favre $\odot,{ }^{1}$ Gregory S. Tucker, ${ }^{1,2}$ Clemens Ritter, ${ }^{3}$ Romain Sibille $\odot,{ }^{2}$ Pascal Manuel, ${ }^{4}$ Matthias D. Frontzek,${ }^{5}$ \\ Markus Kriener ${ }^{0},{ }^{6}$ Lin Yang, ${ }^{1,7}$ Helmuth Berger, ${ }^{8}$ Arnaud Magrez, ${ }^{8}$ Nicola P. M. Casati, ${ }^{9}$ \\ Ivica Živković $\odot{ }^{1, *}$ and Henrik M. Rønnow ${ }^{1, \dagger}$ \\ ${ }^{1}$ Laboratory for Quantum Magnetism, Institute of Physics, Ecole Polytechnique Féderale de Lausanne, CH-1015 Lausanne, Switzerland \\ ${ }^{2}$ Laboratory for Neutron Scattering, Paul Scherrer Institut, CH-5232 Villigen PSI, Switzerland \\ ${ }^{3}$ Institute Laue Langevin, Boîte Postale 156, F-38042, Grenoble, France \\ ${ }^{4}$ ISIS Facility, STFC Rutherford Appleton Laboratory, Oxfordshire OX11 OQX, United Kingdom \\ ${ }^{5}$ Neutron Scattering Division, Oak Ridge National Laboratory, Oak Ridge, Tennessee 37830, USA \\ ${ }^{6}$ RIKEN Center for Emergent Matter Science, Wako 351-0198, Japan \\ ${ }^{7}$ Laboratory of Physics of Complex Matter, Institute of Physics, Ecole Polytechnique Féderale de Lausanne, CH-1015 Lausanne, Switzerland \\ ${ }^{8}$ Crystal Growth Facility, Ecole Polytechnique Fédérale de Lausanne, Lausanne CH-1015, Switzerland \\ ${ }^{9}$ Swiss Light Source, Paul Scherrer Institut, CH-5232 Villigen PSI, Switzerland
}

(Received 24 February 2020; accepted 8 July 2020; published 18 September 2020)

\begin{abstract}
We report magnetic properties of a $3 d^{9}\left(\mathrm{Cu}^{2+}\right)$ magnetic insulator $\mathrm{Cu}_{2} \mathrm{OSO}_{4}$ measured on both powder and single crystal. The magnetic atoms of this compound form layers whose geometry can be described either as a system of chains coupled through dimers or as a kagome lattice where every third spin is replaced by a dimer. Specific heat and DC susceptibility show a magnetic transition at $20 \mathrm{~K}$, which is also confirmed by neutron scattering. Magnetic entropy extracted from the specific heat data is consistent with an $S=1 / 2$ degree of freedom per $\mathrm{Cu}^{2+}$, and so is the effective moment extracted from DC susceptibility. The ground state has been identified by means of neutron diffraction on both powder and single crystal and corresponds to an $\sim 120^{\circ}$ spin structure in which ferromagnetic intradimer alignment results in a net ferrimagnetic moment. No evidence is found for a change in lattice symmetry down to $2 \mathrm{~K}$. Our results suggest that $\mathrm{Cu}_{2} \mathrm{OSO}_{4}$ represents a type of model lattice with frustrated interactions where interplay between magnetic order, thermal and quantum fluctuations can be explored.
\end{abstract}

DOI: 10.1103/PhysRevB.102.094422

\section{INTRODUCTION}

Materials with antiferromagnetic Heisenberg interactions between spins on a triangular lattice [1-3] inherently exhibit large frustration, resulting in many similar energy states giving rise to novel behavior. In practice, there exist several ways to build a full lattice from triangular motifs. The notion of quantum spin liquid was introduced in the form of the resonating valence bond as a potential ground state of the simple triangular lattice $[4,5]$ system. Later developments evidenced that both classical and quantum ground states of the triangular lattice actually displayed long-range order in the so-called $120^{\circ}$ configuration [6]. Among an intense search for quantum spin liquids in models and materials [7,8], considerable attention has been devoted experimentally [9-12] and theoretically [13-15] to the so-called kagome lattice, which is obtained by removing $1 / 4$ of the spins from a triangular lattice, leading to a lower connectivity of four nearest neighbors instead of six, which enhances fluctuations. It has been argued theoretically that the $S=1 / 2$ kagome Heisenberg antiferromagnet shows a spin-liquid ground state $[13,16,17]$, and it is still debated whether the resulting states should be gapped or not [18-20].

\footnotetext{
*ivica.zivkovic@epfl.ch
}

†henrik.ronnow@epfl.ch
Many experimental attempts have been made to find compounds hosting this rich kagome physics [21-25]. Herbertsmithite [26] remains one of the best candidates. Many other candidates exhibited deviations from the kagome model, such as magnetic interactions that go beyond nearest neighbor [27], non-negligible out-of-plane coupling, a significant antisymmetric Dzyaloshinskii-Moriya (DM) interaction [28,29], structural distortions, and chemical disorder. These perturbations lift the degeneracy of an ideal spin liquid and drive the system towards long-range magnetic order.

A major question that currently has no clear answer is to what extent quantum spin liquid properties can survive and coexist with ordered states. This question extends beyond kagome-related systems [30]. It is therefore interesting to study triangle-containing lattice geometries other than the pure triangular and kagome lattices.

In this context we present the study of $\mathrm{Cu}_{2} \mathrm{OSO}_{4}$ [31], which hosts a quasi-two-dimensional (quasi-2D) lattice built from triangular motifs. One way to describe the lattice is that of a kagome lattice with one third of the sites replaced by $S=1 /$ pairs (dimers). Another way to characterize it would be chains coupled through spin pairs in a triangular frustrated pattern. As will be presented below, the spin pair appears to be ferromagnetically aligned, such that an effective model for the system could be described as a kagome lattice with $S=1 / 2$ on $2 / 3$ of the sites and $S=1$ on $1 / 3$ of the 
sites. No single-crystal growth of $\mathrm{Cu}_{2} \mathrm{OSO}_{4}$ has previously been reported [32]. Studies of powder samples of $\mathrm{Cu}_{2} \mathrm{OSO}_{4}$ $[33,34]$ reported the DC susceptibility of the compound and showed evidence of a transition to a magnetically long-rangeordered state at $20 \mathrm{~K}$. The DM interaction in the sample was estimated by means of electron spin resonance [35]. From bulk measurements, a noncollinear antiferromagnetic ground state was proposed [36]. Here we report single-crystal growth and the details of the ground state of $\mathrm{Cu}_{2} \mathrm{OSO}_{4}$, derived from DC susceptibility, magnetization, heat capacity, and $\mathrm{x}$-ray and neutron scattering.

\section{EXPERIMENTAL DETAILS}

Single crystals of $\mathrm{Cu}_{2} \mathrm{OSO}_{4}$ were grown by chemical vapor transport. High quality $\mathrm{Cu}_{2} \mathrm{OSO}_{4}$ powder was synthesized using anhydrous $\mathrm{CuSO}_{4}$ as a source in a quartz crucible placed in the center of a muffle furnace and heated at $740{ }^{\circ} \mathrm{C}$. Two different transport agents were placed in a quartz ampoule at room temperature, $\mathrm{Cl}_{2}$ and $\mathrm{NiBr}_{2}$, along with a portion of the $\mathrm{Cu}_{2} \mathrm{OSO}_{4}$ powder. The ampoules were placed in a two-zone gradient furnace. The best charge and growth-zone temperatures were $650{ }^{\circ} \mathrm{C}$ and $550{ }^{\circ} \mathrm{C}$, respectively. After 5 weeks, several dark-brown, semitransparent crystals were obtained. The typical dimensions of the crystals are $4 \times 2 \times 0.5 \mathrm{~mm}^{3}$. On most of the crystals the $b$ axis can be identified as an edge, and most of them also present a (001) facet.

Specific heat was measured using a physical properties measurement system (PPMS; Quantum Design), and magnetization was measured using both the PPMS and a magnetic properties measurement system (Quantum Design). Neutron diffraction experiments were performed on the Wide Angle Neutron Diffractometer, ZEBRA, D20, and WISH beamlines at the Oak Ridge National Laboratory, Paul Scherrer Institut (PSI), Institute Laue Langevin, and ISIS, respectively. The measurement on D20 was done using the high-resolution option with a takeoff angle of $90^{\circ}$ and a wavelength of 2.41 $\AA$ A. Four-hour measurements were taken at $1.5 \mathrm{~K}$ and at $30 \mathrm{~K}$, while the temperature ramp was done between 1.5 and $30 \mathrm{~K}$ using 30-min runs with about $0.45 \mathrm{~K}$ between consecutive runs. Synchrotron X-ray diffraction was performed at the MSX04SA beamline [37] at Swiss Light Source (SLS), PSI. For powder diffraction, crushed single crystals were used to minimize impurities.

\section{RESULTS}

\section{A. X-ray diffraction and crystal structure}

According to a previous study [31], the compound belongs to the monoclinic space group $C 2 / m$ with lattice parameters $a=9.355(10) \AA, b=6.312(5) \AA, c=7.628(5) \AA, \alpha=\gamma=$ $90^{\circ}$, and $\beta=122.29^{\circ}$.

To determine the temperature dependence of the nuclear structure, we performed temperature-dependent x-ray diffraction on a powder sample. The temperature dependence of lattice parameters was extracted from Le Bail fits (LBFs) and is shown in Fig. 1, along with the unit cell volume. The overall thermal contraction of the lattice has been observed down to $50 \mathrm{~K}$, with a minimum of lattice parameters $a$ and $b$ around $60 \mathrm{~K}$. This nonmonotonic temperature dependence

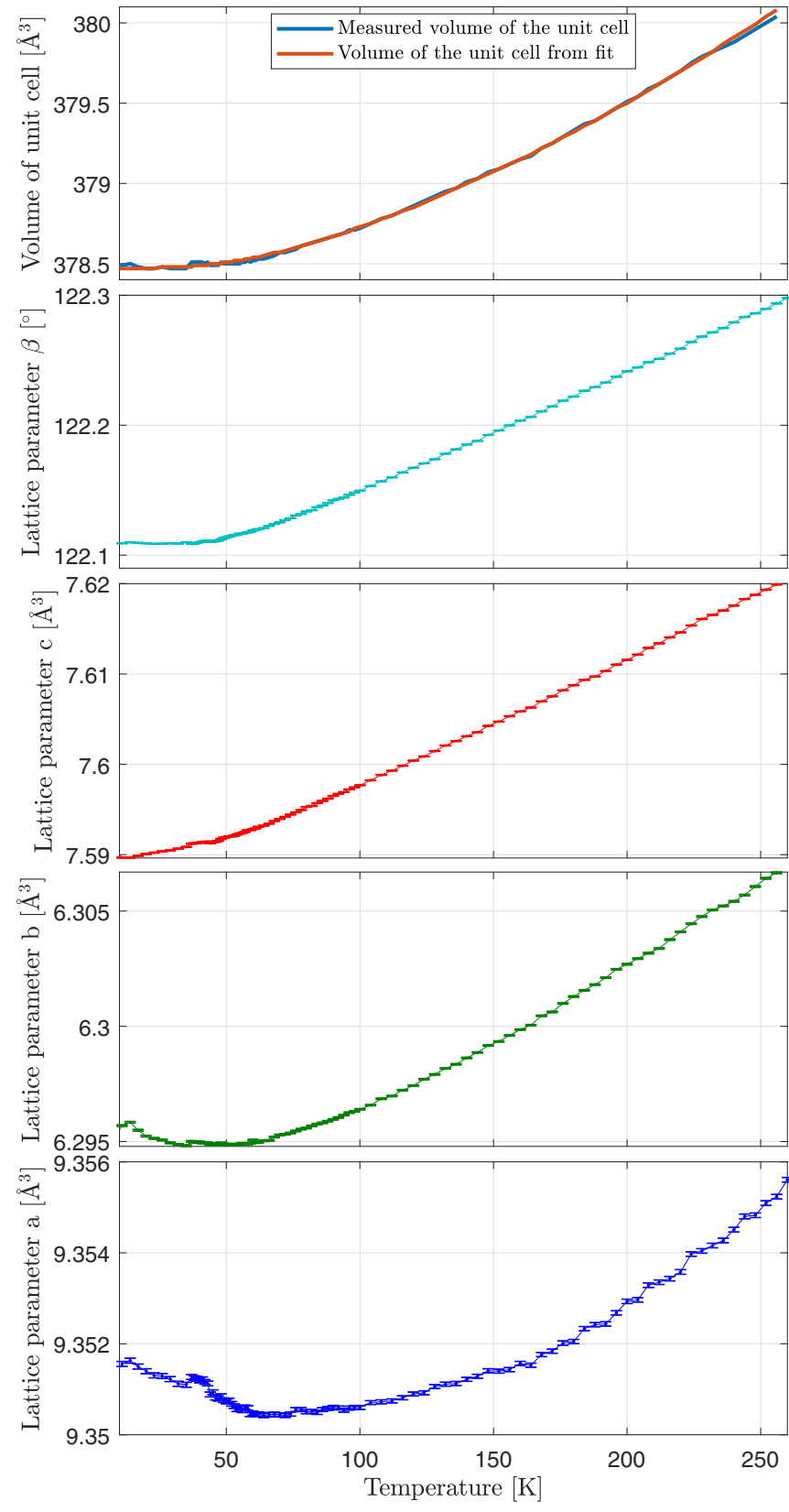

FIG. 1. Change in lattice parameters and unit cell volume obtained from Le Bail fitting of powder x-ray diffraction data.

could be due to the onset of magnetic correlations coupling to the lattice. Similar magnetoelastic coupling is observed below the ordering temperature (Fig. 2).

The crystal structure of $\mathrm{Cu}_{2} \mathrm{OSO}_{4}$ gives rise to an interesting magnetic lattice. The copper atoms occupy two inequivalent positions. They are arranged in the $a b$ plane, with atoms on copper site $1\left(\mathrm{Cu}_{1}\right)$ exactly in the plane, while atoms of copper site $2\left(\mathrm{Cu}_{2}\right)$ are positioned symmetrically above and below the plane. Figure 3(a) shows how the planes are then interconnected through $\mathrm{SO}_{4}$ tetrahedra [38]. The arrangement of $\mathrm{Cu}_{1}$ and $\mathrm{Cu}_{2}$ ions in the quasi-2D planes can be described either as $\mathrm{Cu}_{1}$ chains along the crystallographic $b$ axis, interconnected by $\mathrm{Cu}_{2}$ dimers in a frustrated zigzag pattern, or as a kagome lattice with one third of the sites 


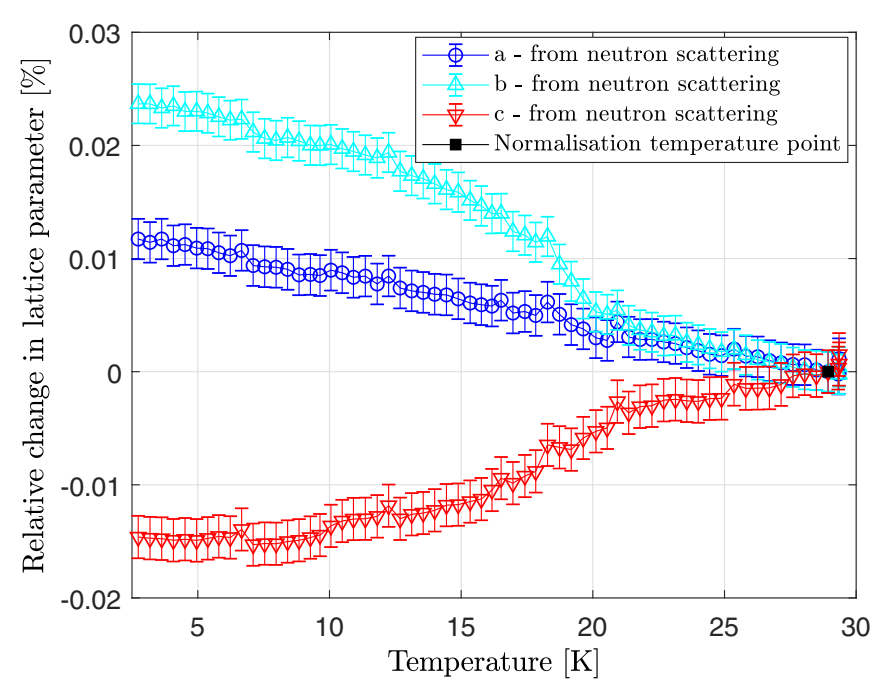

FIG. 2. Relative changes in lattice parameters obtained from neutron diffraction. The data have been normalized to data measured at $29 \mathrm{~K}$ (black square).

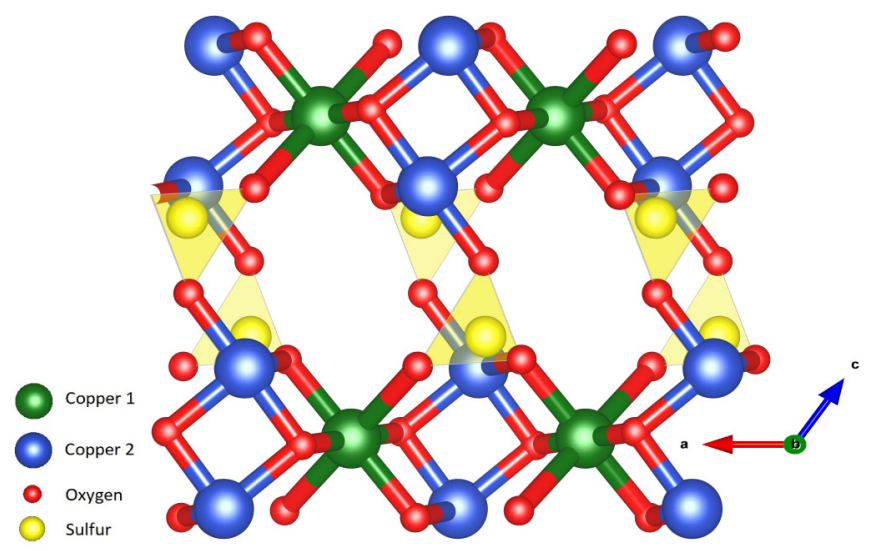

(a)

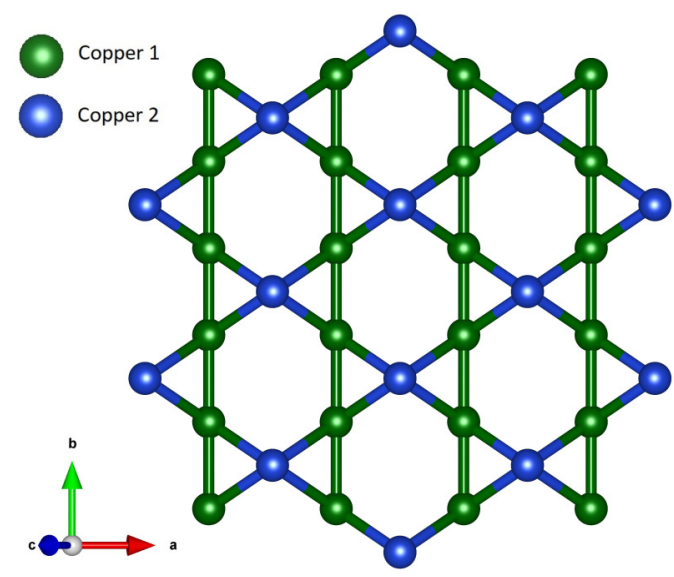

(b)

FIG. 3. $\mathrm{Cu}_{2} \mathrm{OSO}_{4}$ structure obtained from x-ray diffraction. (a) View from the $b$ axis, showing a slice of the kagome-like planes formed by copper atoms in $\mathrm{Cu}_{2} \mathrm{OSO}_{4}$, highlighting the $\mathrm{SO}_{4}$ tertrahedra connecting the copper planes. (b) Kagome-like planes formed by copper atoms in $\mathrm{Cu}_{2} \mathrm{OSO}_{4}$ in the $a b$ plane. The two colors correspond to two inequivalent copper sites.

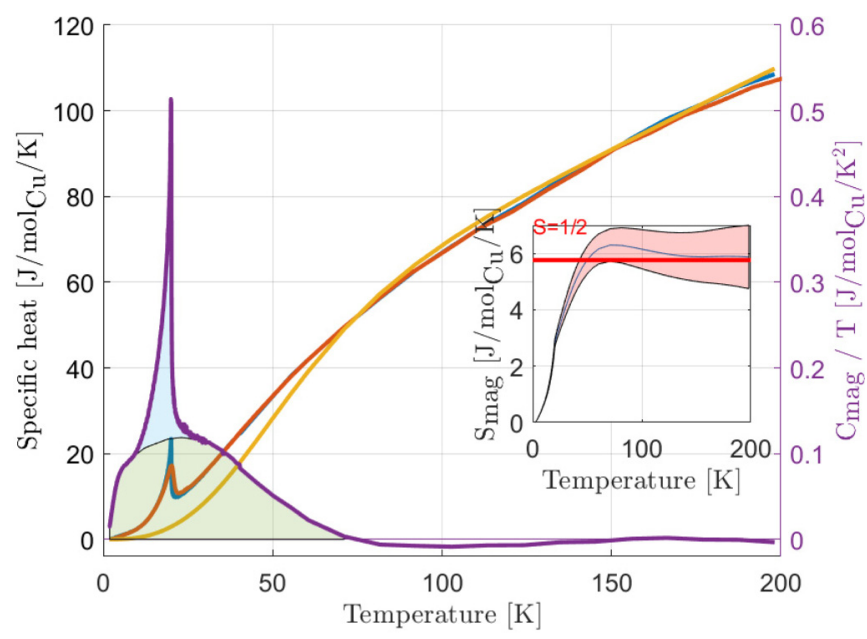

FIG. 4. Specific heat $C_{p}$ as a function of temperature in zero field (blue) and $9 \mathrm{~T}$ (red). The yellow line is the fitted lattice contribution. The purple curve (right vertical axis) shows the magnetic part of the specific heat $C_{\mathrm{mag}} / T$. The blue and green shaded areas represent the estimated $C_{m a g} / T$ that contributes, respectively, to the phase transition and short-range correlations. The inset presents the magnetic entropy, $S_{\mathrm{mag}}(T)=\int C_{\mathrm{mag}}(T) / T d T$. Red shading shows the confidence interval.

replaced by dimers, or it can be viewed as $\mathrm{Cu}_{1}$ chains coupled through $\mathrm{Cu}_{2}$ dimers. Figure 3(b) illustrates how viewing the magnetic layers rotated $8.5^{\circ}$ around the $b$-axis plane overlies the $\mathrm{Cu}_{2}$ ions, resulting in the familiar kagome motif. Figure 9 below shows the magnetic lattice viewed directly along $c^{*}$.

\section{B. Specific heat}

The specific heat $C_{p}$ measured from 2 to $200 \mathrm{~K}$ in zero field and in a magnetic field of $9 \mathrm{~T}$ is shown in Fig. $4 . C_{p}$ above $70 \mathrm{~K}$ for both fields is essentially the same, increasing monotonically with increasing temperature. In zero field a pronounced peak is found at $20 \mathrm{~K}$, corresponding to a transition into a magnetically long-range-ordered phase, as evidenced by other experimental measurements discussed in later sections. The peak shifts to slightly higher temperature at $9 \mathrm{~T}$.

In order to extract the magnetic part of the specific heat $C_{\text {mag }}$ and to deduce the corresponding entropy $\mathrm{S}_{\mathrm{mag}}$, we simulate the lattice contribution from the high-temperature data by taking into account one Debye $\left(C_{D}\right)$ and several Einstein $\left(C_{E, i}\right)$ contributions. We use a combined fit to describe $C_{p}$ and the volume of the unit cell obtained from x-ray diffraction by a phonon (lattice only) model. Similar to what was done in $[39,40]$, the lattice contribution to the specific heat is given by $C_{p}=C_{D}+\sum_{i} C_{E, i}$, with

$$
C_{D}=9 n_{D} R\left(\frac{T}{\Theta_{D}}\right)^{3} \int_{0}^{\Theta_{D} / T} \frac{x^{4} e^{x}}{\left(e^{x}-1\right)^{2}} d x
$$

and

$$
C_{E}=3 n_{E} R \frac{y^{2} e^{y}}{\left(e^{y}-1\right)^{2}}, \quad y \equiv \Theta_{E} / T,
$$

where $R$ denotes the gas constant and $\Theta_{D}$ and $\Theta_{E}$ are the Debye and Einstein temperatures, respectively. The sum $n_{D}+$ 
$n_{E}$ is the total number of atoms per formula unit. The volume of the unit cell has been fitted together with the specific heat using the Debye and Einstein contributions to the internal energy. The volume of the unit cell is related to the internal energy by [41-43]

$$
V(T)=\gamma U(T) / K_{0}+V_{0},
$$

where $V_{0}$ is the cell volume at $T=0 \mathrm{~K}, K_{0}$ is the bulk modulus, and $\gamma$ is the Grüneisen parameter. $U(T)$ is the internal energy, which can be expressed in terms of the Debye and Einstein approximation as

$$
\begin{gathered}
U(T)=U_{\mathrm{D}}(T)+U_{\mathrm{E}}(T), \\
U_{\mathrm{D}}(T)=9 n_{D} k_{\mathrm{B}} T\left(\frac{T}{\Theta_{\mathrm{D}}}\right)^{3} \int_{0}^{\Theta_{\mathrm{D}} / T} \frac{x^{3}}{e^{x}-1} d x, \\
U_{\mathrm{E}}(T)=\frac{3}{2} k_{\mathrm{B}} \sum_{i} n_{\mathrm{E}, \mathrm{i}} \Theta_{\mathrm{E}, \mathrm{i}} \operatorname{coth}\left(\frac{\Theta_{\mathrm{E}, \mathrm{i}}}{2 T}\right) .
\end{gathered}
$$

The best fit for both sets of data, using one Debye branch and two Einstein branches, yields the characteristic temperatures $\Theta_{D}=171 \mathrm{~K}, \Theta_{E 1}=245 \mathrm{~K}$, and $\Theta_{E 2}=939 \mathrm{~K}$, with $n_{D}=1$, $n_{E 1}=3$, and $n_{E 2}=4$. This fit was performed using data for temperatures larger than $70 \mathrm{~K}$. The volume of the unit cell at $0 \mathrm{~K}$ has also been fitted to be $V_{0}=373.65 \AA^{3}$ (Fig. 1).

Figure 4 shows the resulting $C_{\mathrm{mag}} / T$ in zero field as well as the magnetic entropy $S_{\mathrm{mag}}(T)$ obtained by integrating $C_{\mathrm{mag}} / T$ over temperature. The magnetic entropy saturates around $101(19) \%$ of $R \ln 2$ per formula unit, which is in agreement with the entropy of a two-level spin-half system. A similar analysis carried out on the 9-T data (not shown) indicates negligible field effects. The magnetic entropy is shared between short-range correlations (Fig. 4, green shading) and phase transition to long-range order (Fig. 4, blue shading). We estimate that $18(5) \%$ of the magnetic entropy contributes to the phase transition.

\section{Susceptibility and magnetization}

The inverse DC susceptibility measured on a single crystal, with a field of $0.1 \mathrm{~T}$, pointing along the three relevant crystallographic directions, $a, b$, and $c^{*}$, is shown in Fig. 5. The high-temperature part of $\chi(T)$ fits the Curie-Weiss law, $\chi(T)=C /\left(T-\Theta_{\mathrm{CW}}\right)+\chi_{0}$, where $\Theta_{\mathrm{CW}}$ is the Curie-Weiss temperature, $C=\mu_{\text {eff }}^{2} / 8$, and $\chi_{0}$ is a temperature-independent diamagnetic and background term. $\mu_{\text {eff }}=g \sqrt{S(S+1)}$ is the effective magnetic moment, which is temperature independent. For a spin-half system with a Landé $g$ factor of 2 the effective magnetic moment has a value of approximately $1.7 \mu_{\mathrm{B}}$. Deviations from this value can be explained by either a slightly different spin state or a $g$ factor different from 2 , which is also possibly anisotropic. The fitted effective moments, $\Theta_{\mathrm{CW}}$ and $\chi_{0}$, are given in Table I, and the corresponding fits can be observed in Fig. 5. The Curie-Weiss temperature is isotropic within error bars and negative, which indicates dominating antiferromagnetic interactions. The $g$ tensor is slightly anisotropic with the easy axis along $b$ and the hard axis along $a$. Below $20 \mathrm{~K}$ the susceptibility steeply increases, indicating a magnetic transition into a state with a ferromagnetic component. Zero-field-cooled-field-cooled

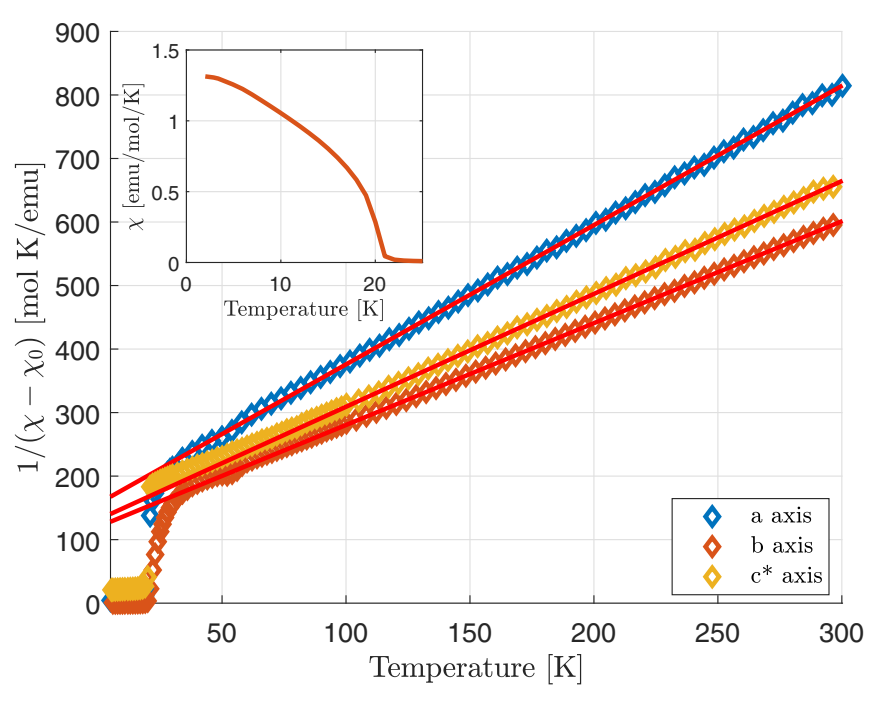

FIG. 5. Curie-Weiss fits of the high-temperature susceptibility data, along the $a$ axis(blue), $b$ axis (red), and $c^{*}$ axis (yellow). The red lines indicate the fit for each direction.

splitting at low temperature is typical of weak ferromagnetic hysteresis. The magnetic transition occurring well below $\Theta_{\mathrm{CW}}$ indicates order is suppressed by fluctuations due to a combination of low dimensionality and frustration.

Figure 6 shows difference in the field-cooled and zerofield-cooled DC magnetic susceptibility measured along the $b$ axis for different values of the measurement field: $H=5 \mathrm{mT}$, $H=10 \mathrm{mT}, H=20 \mathrm{mT}, H=50 \mathrm{mT}$.

The susceptibility along the other two crystallographic directions (not shown; $a$ and $c^{*}$ ) shows a similar temperature dependence, but with a much smaller value reached at low temperatures. We conclude that the susceptibility grows only along the $b$ direction and that the small signal in the $a$ and $c^{*}$ measurements is due to a small misalignment of the crystal.

Figure 7 shows the isothermal magnetization curves for fields parallel to the $b$ axis. For temperatures higher than $20 \mathrm{~K}$, the curves are linear, indicating a paramagnetic state. Below $20 \mathrm{~K}$, a small hysteresis opens. The saturation field increases when the temperature decreases and reaches $40 \mathrm{mT}$ at $2 \mathrm{~K}$. No similar hysteresis opens when applying the field along the $c^{*}$ axis or along the $a$ axis (not shown). This is another indication of a ferrimagnetic ground state with a moment $\simeq 1 / 4 \mu_{B}$ pointing along the $b$ axis. The hysteresis curve is slightly asymmetric and displays a slow time dependence. This exchange-bias-related phenomenon could reflect that the ferrimagnetism is the result of coupling ferromagnetic and antiferromagnetic subunits.

TABLE I. Results of Curie-Weiss fits for measurements along $a$, $b$, and $c^{*}$.

\begin{tabular}{lccc}
\hline \hline Direction & $\Theta_{\mathrm{CW}}(\mathrm{K})$ & $\chi_{0}$ & $\mu_{\text {eff }}\left(\right.$ units of $\left.\mu_{B}\right)$ \\
\hline$a$ & $-71(4)$ & $5.4 \times 10^{-5}$ & $1.91(25)$ \\
$b$ & $-75(1)$ & $4.7 \times 10^{-4}$ & $2.23(25)$ \\
$c^{*}$ & $-70(1)$ & $-7.6 \times 10^{-5}$ & $2.11(11)$ \\
\hline
\end{tabular}




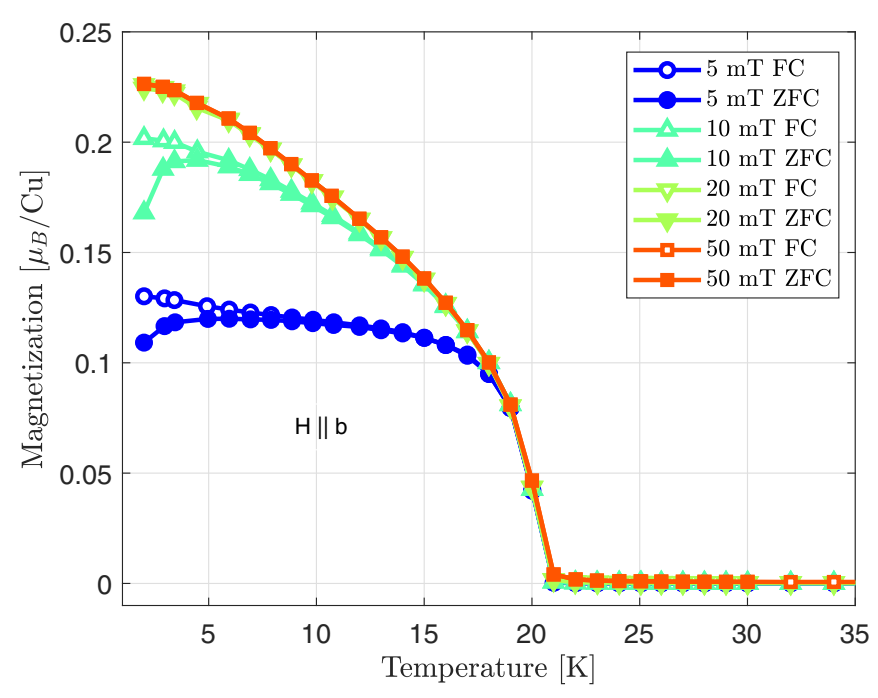

FIG. 6. Magnetization measured on a single crystal with field along the $b$ axis highlighting the field-cooled-zero-field-cooled separation in low fields. The strongest separation occurs at $5 \mathrm{mT}$ and decreases with field.

\section{Neutron diffraction}

To determine the magnetic structure, neutron diffraction measurements were performed on powder samples. Figure 8 shows in blue the data measured at $1.5 \mathrm{~K}$. The magnetic scattering has been separated by measuring a powder diffraction pattern above the magnetic transition at $30 \mathrm{~K}$. The subtraction of the nuclear data from the base temperature data is displayed in red in Fig. 8. Some up-down features appear due to lattice contraction, changing the Bragg peak positions.

Every magnetic contribution is located at a nuclear Bragg peak. This indicates that the magnetic propagation vector is $k=(000)$. Magnetic symmetry analysis was done for this propagation vector using the program BASIREPS [44-46] for Wykoff sites $42\left(\mathrm{Cu}_{1}\right)$ and $4 \mathrm{i}\left(\mathrm{Cu}_{2}\right)$ in space group $C 2 / \mathrm{m}$. Table II lists the allowed irreducible representations (irreps),

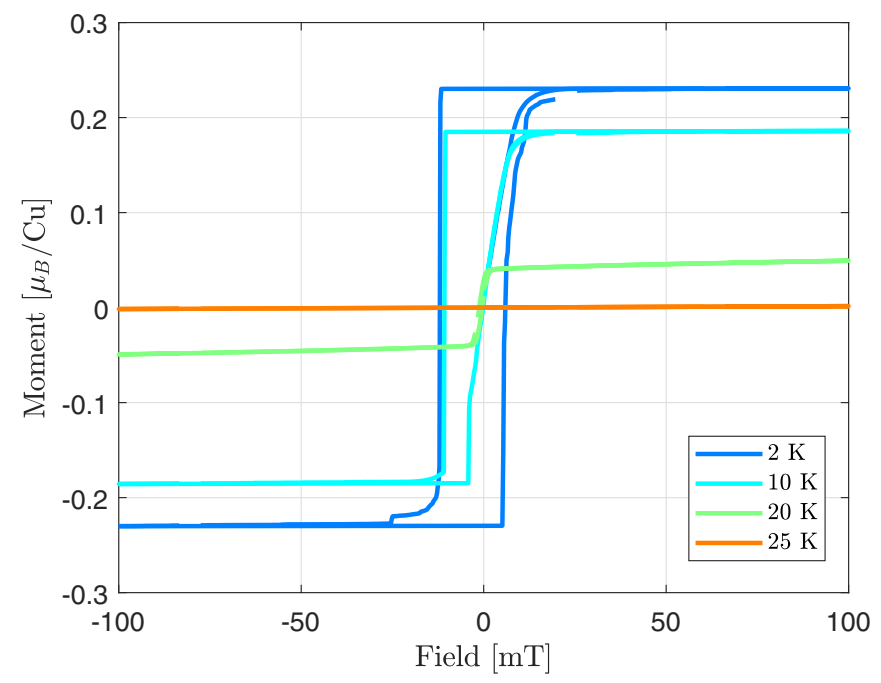

FIG. 7. Isothermal magnetization for $H \| b$ measured on a single crystal.
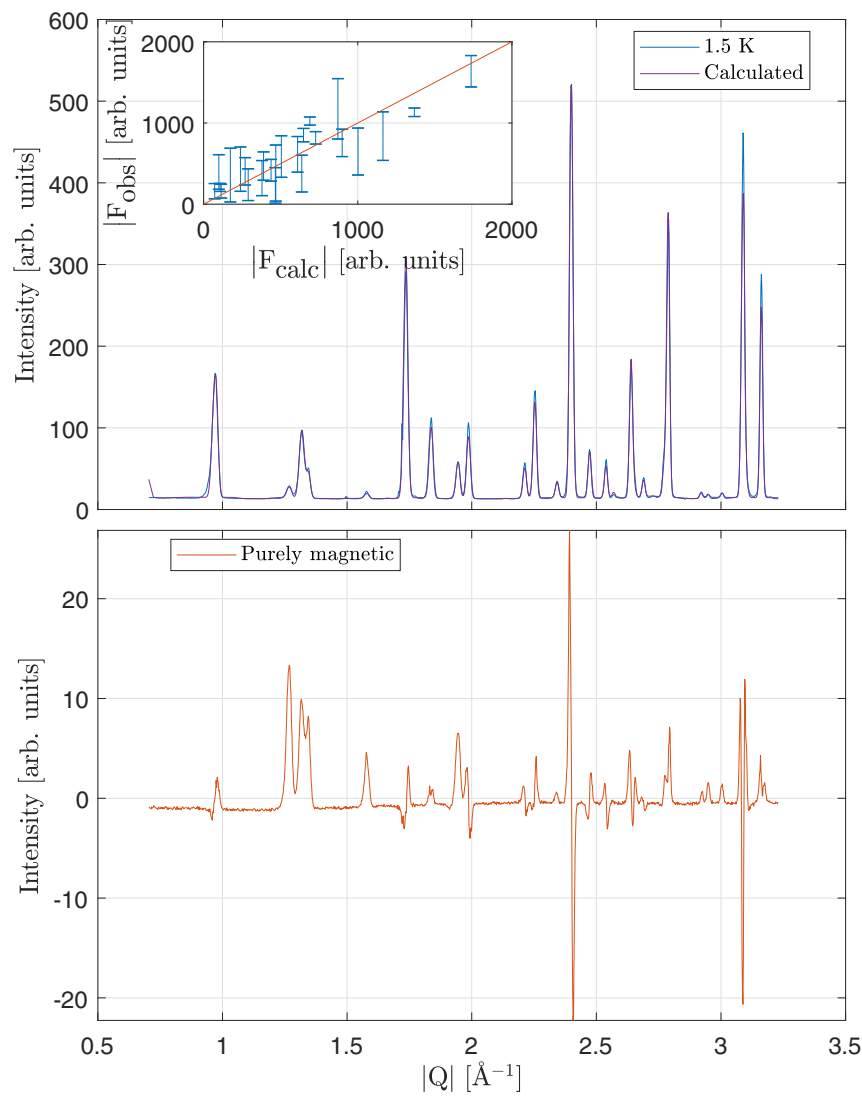

FIG. 8. Refinement of neutron diffraction data from D20: lowesttemperature data measured at $1.5 \mathrm{~K}$ (blue) and result of the fit (purple). The purely magnetic signal (difference between data measured above and below the transition temperature) is shown in red. The inset shows the result of magnetic refinement of the data collected at ZEBRA, with irreps $\Gamma_{1}$ at a base temperature of $1.5 \mathrm{~K}$. The curves display the observed versus the calculated structure factors. The best fit shows good qualitative agreement with the model.

labeled $\Gamma_{1}-\Gamma_{4}$, and their basis vectors, labeled $(u, v, w)$ for site 1 and $(r, s, t)$ for site 2 , and shows how the magnetic moments on the symmetry-related sites $x, y, z$ and $-x, y,-z$ are constrained and transformed by the different possible irreps.

Refinements were carried out using the FULLPROF suite [44]. The $30 \mathrm{~K}$ data were first refined to solve the nuclear structure. The scale factor obtained in this refinement was then fixed and used to refine the purely magnetic scattering of a data set created by subtracting the $30 \mathrm{~K}$ data from the $1.5 \mathrm{~K}$

TABLE II. Basis functions of irreducible representations $\Gamma_{v}$ for $k=(000)$. Only the real components are presented because the imaginary part is zero. The two equivalent copper sites are related through the indicated transformations.

\begin{tabular}{lcccc}
\hline \hline & $\mathrm{Cu}_{1}: x, y, z$ & $\mathrm{Cu}_{1}:-x, y,-z$ & $\mathrm{Cu}_{2}: x, y, z$ & $\mathrm{Cu}_{2}:-x, y,-z$ \\
\hline$\Gamma_{1}$ & $(u, v, w)$ & $(-u, v,-w)$ & $(0 s 0)$ & $(0 s 0)$ \\
$\Gamma_{2}$ & & & $(r 0 t)$ & $(-r 0-t)$ \\
$\Gamma_{3}$ & $(u, v, w)$ & $(u,-v, w)$ & $(r 0 t)$ & $(r 0 t)$ \\
$\Gamma_{4}$ & & & $(0 s 0)$ & $(0-s 0)$ \\
\hline \hline
\end{tabular}



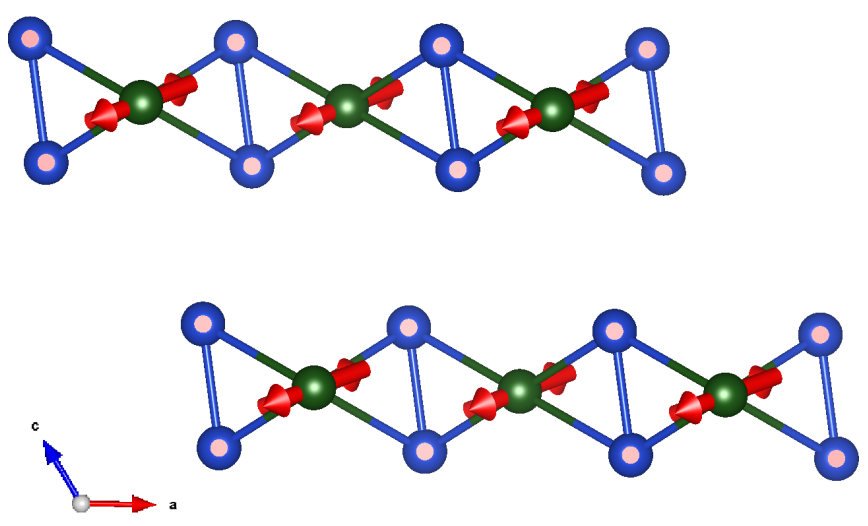

(a)

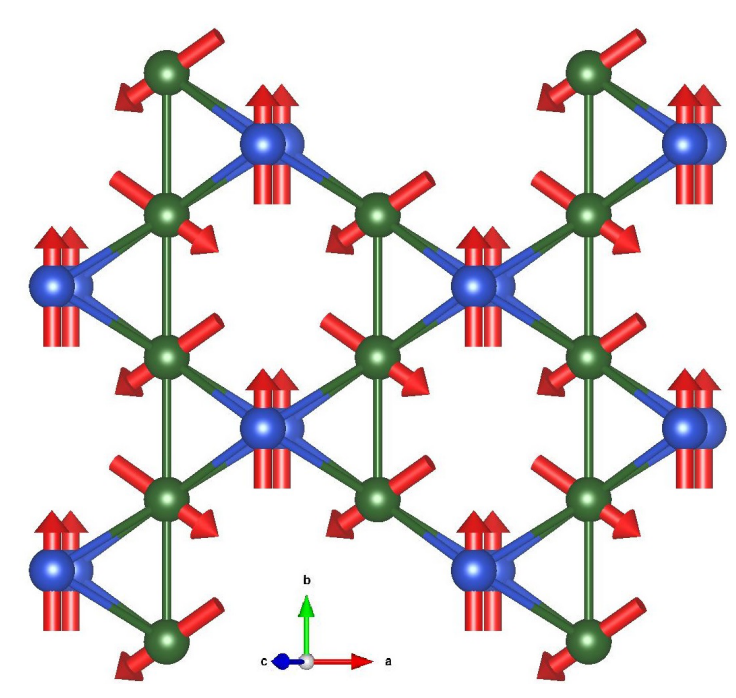

(b)

FIG. 9. Magnetic structure of $\mathrm{Cu}_{2} \mathrm{OSO}_{4}$ refined with irrep $\Gamma_{1}$ on both sites. Copper site 1 is shown in green, while copper site 2 is shown in blue.

data with the same statistics. In doing so, the sensitivity for the magnetic contribution increases strongly, reducing the uncertainties in the determination of the magnetic components.

In addition to the powder diffraction we also carried out a single-crystal neutron diffraction experiment on the fourcircle diffractometer ZEBRA, at SINQ, PSI. The crystal used turned out to present a strong mosaicity, which did not enable us to collect intensity at Bragg reflections in a systematic way and in all the directions of reciprocal space. Intensities for a set of 55 reflections were collected at a base temperature of $1.5 \mathrm{~K}$ as well as at $25 \mathrm{~K}$ to solve the nuclear structure. The result of the fit of the experimental data is presented in the inset of Fig. 8 for a choice of irrep $\Gamma_{1}$ on both copper sites.

The best refinement is given by $\Gamma_{1}$ on both inequivalent sites. Figure 8 shows in purple the result of the calculation of the base temperature data using the fit result. Figure 9 shows the associated configuration of magnetic moments. The result of the fit of irreducible representation $\Gamma_{1}$ on both sites is presented in Table III. If the irreps are set to the result obtained by the powder diffraction experiment, then the free parameters in the single-crystal refinement from ZEBRA can be reasonably adjusted and lead to a magnetic structure showing good agreement with the one obtained from the refinement of the powder data. The magnetic structure consists of $\mathrm{Cu}_{1}$ antiferromagnetic chains with noncollinear moments. This is the configuration that a one-dimensional chain would adopt in magnetic field along the chain, with the field strength equal to half the saturation field. The chains are connected by $\mathrm{Cu}_{2}$ dimers. The $\mathrm{Cu}_{2}$ atoms in the dimer are ferromagnetically coupled. The copper atoms in the chains form an approximately $120^{\circ}$ structure with four spins: two $\mathrm{Cu}_{2}$ atoms and two $\mathrm{Cu}_{1}$ atoms belonging to the $\mathrm{Cu}_{1}$ chains. The magnetic moments along the $a$ and $c^{*}$ directions are compensated, and the magnetic structure supports only a net moment along the $b$ axis. Using values obtained from neutron diffraction in Table III, we obtain the following saturation moment along the $b$ axis: $0.23(3) \mu_{\mathrm{B}}$. This moment is made up of a quarter of the sum of the moments of two $\mathrm{Cu}_{1}$ and two $\mathrm{Cu}_{2}$ atoms and is in perfect agreement with the isothermal magnetization shown in Fig. 7.

Temperature-dependent neutron diffraction was performed on a powder sample. Using LBFs, one can extract the associated change in the lattice parameters on the whole measured range. Figure 2 shows the relative change in the lattice parameters, which reflects the onset of magnetic order below $20 \mathrm{~K}$. Figure 10 shows the evolution of the ordered magnetic moments as a function of temperature, obtained from neutron diffraction. Solid lines correspond to power-law fits: $\mu(T) \propto$ $\left(T_{N}-T\right)^{2 \beta}$. The fit of the data yields $T_{N}=19.7(7) \mathrm{K}$, shared by the two sites, and $\beta=0.12$ (1) for site 1 and $\beta=0.13(2)$ for site 2 .

\section{DISCUSSION}

The ordered structure determined from the neutron powder diffraction is fully consistent with exchange paths present in this compound. A close inspection of the structure reveals which superexchange paths are the most relevant. The pathway between two $\mathrm{Cu}_{2}$ ions contains oxygen ions, forming the $\mathrm{Cu}_{2}-\mathrm{O}-\mathrm{Cu}_{2}$ angle of $\sim 93.0^{\circ}$, which favors a ferromagnetic $J_{22}$ according to the Goodenough-Kanamori-Anderson (GKA) rules. On the other hand, the $\mathrm{Cu}_{1}-\mathrm{O}-\mathrm{Cu}_{1}$ angle between two

TABLE III. Result of the fit to magnetic irrep $\Gamma_{1}$. The second row shows results while constraining the moments to the $a b$ plane.

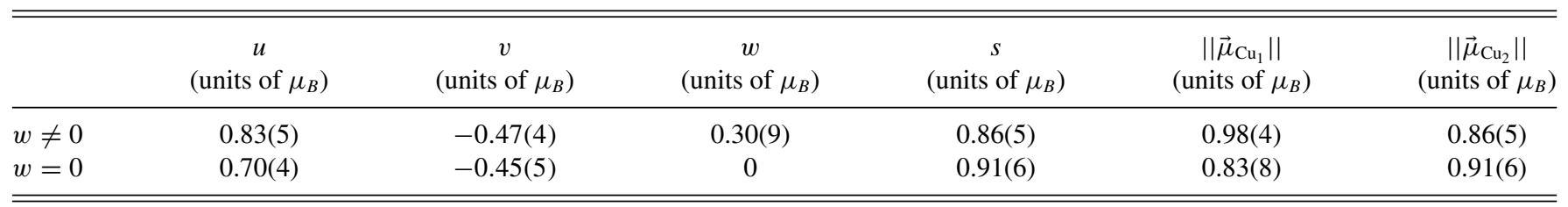




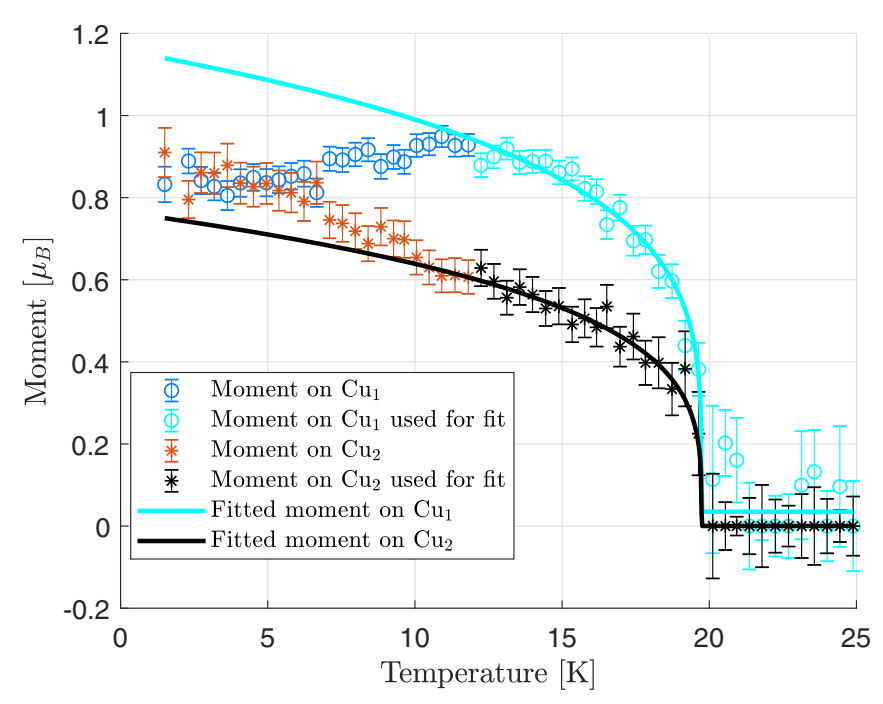

FIG. 10. Temperature dependence of magnetic refinements with irreps $\Gamma_{1}$. The solid lines are a power-law fit to the magnetic moment on each site: $\mu(T) \propto\left(T_{N}-T\right)^{2 \beta}$. The fitting of the power law behavior has been performed over the specified data range (black and cyan).

nearest-neighbor $\mathrm{Cu}_{1}$ atoms is $114.9^{\circ}$. This falls well within the range of values predicted by GKA rules to favor antiferromagnetic $J_{11}$ interactions. For the coupling between two inequivalent copper sites there are two distinct superexchange paths, one forming a $\mathrm{Cu}_{1} \mathrm{OCu}_{2}$ angle of $104.9^{\circ}$, while the other one is $117.3^{\circ}$. Such values are again consistent with antiferromagnetic couplings ( $J_{21 a}$ and $J_{21 b}$, respectively).

Additional exchange pathways exist and involve sulfur tetrahedra, more precisely, $\mathrm{Cu}-\mathrm{O}-\mathrm{O}-\mathrm{Cu}$ super-superexchange interactions. They provide a coupling between $\mathrm{Cu}_{2}$ dimers as well as an overall interplanar coupling which eventually leads to the observed long-range order. It is a nontrivial task to estimate their relative strength to superexchange in-plane coupling. Future inelastic neutron scattering experiments and modeling of dispersion relations could give us an indication of which interactions are the most relevant for the long-range order in $\mathrm{Cu}_{2}-\mathrm{O}-\mathrm{Cu}_{2}$.

As a final note, an antisymmetric DM interaction has been indicated to exist in this compound [35], compatible with crystal symmetry. Its magnitude has been estimated to be $D \sim 7 \mathrm{~K}$. If we take $\theta_{C W} \sim 70 \mathrm{~K}$ as an estimate for $J$, we arrive at $D / J \gtrsim 0.1$. For the kagome lattice, DM interactions have been predicted to cause a quantum phase transition stabilizing $120^{\circ}$ order above $D_{c}=0.1 J$ [28,47]. Given the similar relative order of $D$ in $\mathrm{Cu}_{2} \mathrm{OSO}_{4}$, it is possible that this interaction is partly responsible for the $120^{\circ}$ order, and as a consequence, $\mathrm{Cu}_{2} \mathrm{OSO}_{4}$ may be close to a quantum phase transition, which could possibly be reached by tuning the system through pressure or chemical substitution.

\section{CONCLUSION}

In summary, we have presented a detailed study of the magnetic properties of $\mathrm{Cu}_{2} \mathrm{OSO}_{4}$. We confirmed that the structure of $\mathrm{Cu}_{2} \mathrm{OSO}_{4}$ does not change upon cooling and that the ground state corresponds to an $S=1 / 2$ system. The Curie-Weiss temperatures give an idea of the order of magnitude of the magnetic couplings involved in the system, $70 \mathrm{~K}$; however, the system undergoes a second-order magnetic phase transition to a magnetically long range ordered state only below $20 \mathrm{~K}$. Neutron scattering reveals that the ground state corresponds roughly to the $120^{\circ}$ order. The fact that this specific magnetic structure turns out to be the ground state, even though one third of the sites in the kagome-like lattice are replaced by ferromagnetic dimers, is interesting. The rather small entropy linked to the transition suggest that there might be interesting dynamics related to the formation of ferromagnetic pairs and $120^{\circ}$ triangles and subsequent alignment of these units. We hope this study will stimulate further theoretical and experimental studies of the dynamics in this triangular motif model compound.

\section{ACKNOWLEDGMENTS}

V.Y.F. thanks P. Babkevich for his help with the refinement of diffraction data and F. Mila for stimulating discussions. We acknowledge the Paul Scherrer Institut, Villigen, Switzerland, for provision of synchrotron radiation beam time at beamline MS-X04SA of the SLS. This work is partially based on experiments performed at the Swiss spallation neutron source SINQ, Paul Scherrer Institute, Villigen, Switzerland, at the STFC ISIS Facility [48], and at the Institut Laue-Langevin [49]. A portion of this research used resources at the High Flux Isotope Reactor, a DOE Office of Science User Facility operated by the Oak Ridge National Laboratory. This work was supported by the Swiss National Science Foundation (SNSF), Grant No. 188648.
[1] A. Olariu, P. Mendels, F. Bert, B. G. Ueland, P. Schiffer, R. F. Berger, and R. J. Cava, Unconventional Dynamics in Triangular Heisenberg Antiferromagnet $\mathrm{NaCrO}_{2}$, Phys. Rev. Lett. 97, 167203 (2006).

[2] Y. Shirata, H. Tanaka, A. Matsuo, and K. Kindo, Experimental Realization of a Spin-1/2 Triangular-Lattice Heisenberg Antiferromagnet, Phys. Rev. Lett. 108, 057205 (2012).

[3] Y. Kojima, M. Watanabe, N. Kurita, H. Tanaka, A. Matsuo, K. Kindo, and M. Avdeev, Quantum magnetic properties of the spin-1/2 triangular-lattice antiferromagnet $\mathrm{Ba}_{2} \mathrm{La}_{2} \mathrm{CoTe}_{2} \mathrm{O}_{12}$, Phys. Rev. B 98, 174406 (2018).
[4] P. Anderson, Resonating valence bonds: A new kind of insulator? Mater. Res. Bull. 8, 153 (1973).

[5] P. Fazekas and P. W. Anderson, On the ground state properties of the anisotropic triangular antiferromagnet, Philos. Mag. 30, 423 (1974).

[6] M. Collins and O. Petrenko, Triangular antiferromagnets, Can. J. Phys. 75, 605 (1997).

[7] F. Ferrari, A. Parola, S. Sorella, and F. Becca, Dynamical structure factor of the $J_{1}-J_{2}$ Heisenberg model in one dimension: The variational Monte Carlo approach, Phys. Rev. B 97, 235103 (2018). 
[8] O. Mustonen, S. Vasala, E. Sadrollahi, K. Schmidt, C. Baines, H. Walker, I. Terasaki, F. Litterst, E. Baggio-Saitovitch, and M. Karppinen, Spin-liquid-like state in a spin-1/2 square-lattice antiferromagnet perovskite induced by $\mathrm{d} 10-\mathrm{d} 0$ cation mixing, Nat. Commun. 9, 1085 (2018).

[9] M. P. Shores, E. A. Nytko, B. M. Bartlett, and D. G. Nocera, A Structurally Perfect $S=1 / 2$ Kagomé Antiferromagnet, J. Am. Chem. Soc. 127, 13462 (2005).

[10] P. Mendels and F. Bert, Quantum kagome frustrated antiferromagnets: One route to quantum spin liquids, C. R. Phys. 17, 455 (2016).

[11] J. S. Helton, K. Matan, M. P. Shores, E. A. Nytko, B. M. Bartlett, Y. Yoshida, Y. Takano, A. Suslov, Y. Qiu, J.-H. Chung, D. G. Nocera, and Y. S. Lee, Spin Dynamics of the Spin-1/2 Kagome Lattice Antiferromagnet $\mathrm{ZnCu}_{3}(\mathrm{OH})_{6} \mathrm{Cl}_{2}$, Phys. Rev. Lett. 98, 107204 (2007).

[12] A. Zorko, S. Nellutla, J. van Tol, L. C. Brunel, F. Bert, F. Duc, J.-C. Trombe, M. A. de Vries, A. Harrison, and P. Mendels, Dzyaloshinsky-Moriya Anisotropy in the Spin-1/2 Kagome Compound $\mathrm{ZnCu}_{3}(\mathrm{OH})_{6} \mathrm{Cl}_{2}$, Phys. Rev. Lett. 101, 026405 (2008).

[13] S. Depenbrock, I. P. McCulloch, and U. Schollwock, Nature of the Spin-Liquid Ground State of the $S=1 / 2$ Heisenberg Model on the Kagome Lattice, Phys. Rev. Lett. 109, 067201 (2012).

[14] F. Mila, Low-Energy Sector of the $S=1 / 2$ Kagome Antiferromagnet, Phys. Rev. Lett. 81, 2356 (1998).

[15] R. R. P. Singh and D. A. Huse, Ground state of the spin-1/2 kagome-lattice Heisenberg antiferromagnet, Phys. Rev. B 76, 180407(R) (2007).

[16] S. Yan, D. A. Huse, and S. R. White, Spin-Liquid Ground State of the $S=1 / 2$ Kagome Heisenberg Antiferromagnet, Science 332, 1173 (2011).

[17] M. Hermele, Y. Ran, P. A. Lee, and X.-G. Wen, Properties of an algebraic spin liquid on the kagome lattice, Phys. Rev. B 77, 224413 (2008).

[18] L. Clark, J. C. Orain, F. Bert, M. A. De Vries, F. H. Aidoudi, R. E. Morris, P. Lightfoot, J. S. Lord, M. T. F. Telling, P. Bonville, J. P. Attfield, P. Mendels, and A. Harrison, Gapless Spin Liquid Ground State in the $S=1 / 2$ Vanadium Oxyfluoride Kagome Antiferromagnet $\left[\mathrm{NH}_{4}\right]_{2}\left[\mathrm{C}_{7} \mathrm{H}_{14} \mathrm{~N}\right]\left[\mathrm{V}_{7} \mathrm{O}_{6} \mathrm{~F}_{18}\right]$, Phys. Rev. Lett. 110, 207208 (2013).

[19] Y. Iqbal, F. Becca, S. Sorella, and D. Poilblanc, Gapless spinliquid phase in the kagome spin-1 2 Heisenberg antiferromagnet, Phys. Rev. B 87, 060405 (2013).

[20] M. Fu, T. Imai, T.-H. Han, and Y. S. Lee, Evidence for a gapped spin-liquid ground state in a kagome Heisenberg antiferromagnet, Science 350, 655 (2015).

[21] M. Takano, T. Shinjo, and T. Takada, On the spin arrangement in kagome lattice of antiferromagnetic $\mathrm{KFe}_{3}(\mathrm{OH})_{6}\left(\mathrm{SO}_{4}\right)_{2}$, J. Phys. Soc. Jpn. 30, 1049 (1971).

[22] Y. Okamoto, H. Yoshida, and Z. Hiroi, Vesignieite $\mathrm{BaCu}_{3} \mathrm{~V}_{2} \mathrm{O}_{8}(\mathrm{OH})_{2}$ as a Candidate Spin-1/2 Kagome Antiferromagnet, J. Phys. Soc. Jpn. 78, 033701 (2009).

[23] T. H. Han, J. S. Helton, S. Chu, A. Prodi, D. K. Singh, C. Mazzoli, P. Müller, D. G. Nocera, and Y. S. Lee, Synthesis and characterization of single crystals of the spin-1/2 kagome-lattice antiferromagnets $\mathrm{Zn}_{x} \mathrm{Cu}_{4-x}(\mathrm{OH})_{6} \mathrm{Cl}_{2}$, Phys. Rev. B 83, 100402(R) (2011).

[24] B. Martínez, F. Sandiumenge, A. Rouco, A. Labarta, J. Rodríguez-Carvajal, M. Tovar, M. Causa, S. Galí, and X.
Obradors, Magnetic dilution in the strongly frustrated kagome antiferromagnet $\mathrm{SrGa}_{12-x} \mathrm{Cr}_{x} \mathrm{O}_{19}$, Phys. Rev. B 46, 10786 (1992).

[25] Z. Hiroi, M. Hanawa, N. Kobayashi, M. Nohara, H. Takagi, Y. Kato, and M. Takigawa, Spin-1/2 kagome-like lattice in volborthite $\mathrm{Cu}_{3} \mathrm{~V}_{2} \mathrm{O}_{7}(\mathrm{OH})_{2} 2 \mathrm{H}_{2} \mathrm{O}$, J. Phys. Soc. Jpn. 70, 3377 (2001)

[26] P. Mendels and F. Bert, Quantum Kagome Antiferromagnet $\mathrm{ZnCu}_{3}(\mathrm{OH})_{6} \mathrm{Cl}_{2}$, J. Phys. Soc. Jpn. 79, 011001 (2010).

[27] M. Wolf and K. D. Schotte, Ising model with competing nextnearest-neighbour interactions on the kagome lattice, J. Phys. A 21, 2195 (1988).

[28] O. Cépas, C. M. Fong, P. W. Leung, and C. Lhuillier, Quantum phase transition induced by Dzyaloshinskii-Moriya interactions in the kagome antiferromagnet, Phys. Rev. B 78, 140405(R) (2008).

[29] Y. Huh, L. Fritz, and S. Sachdev, Quantum criticality of the kagome antiferromagnet with Dzyaloshinskii-Moriya interactions, Phys. Rev. B 81, 144432 (2010).

[30] B. Dalla Piazza, M. Mourigal, N. B. Christensen, G. J. Nilsen, P. Tregenna-Piggott, T. G. Perring, M. Enderle, D. F. McMorrow, D. A. Ivanov, and H. M. Rønnow, Fractional excitations in the square-lattice quantum antiferromagnet, Nat. Phys. 11, 62 (2015).

[31] E. Flügel-Kahler, Die Kristallstruktur von Dolerophanit, $\mathrm{Cu}_{2} \mathrm{O}\left(\mathrm{SO}_{4}\right)$, Acta Crystallogr. 16, 1009 (1963).

[32] L. Bald, M. Spiess, R. Gruehn, and T. Kohlmann, Beitrage zum thermischen Verhalten von Sulfaten. VI. Zum chemischen Transport von $\mathrm{CuSO}_{4}, \mathrm{Cu}_{2} \mathrm{OSO}_{4}$ und $\mathrm{CuO}, \mathrm{Z}$. Anorg. Allg. Chem. 498, 153 (1983).

[33] M. Belaiche, M. Drillon, J. Aride, A. Boukharis, T. Biaz, and P. Legoll, Application du modele Heisenberg a la chaine ferrimagnetique d ions $\mathrm{Cu}(\mathrm{II})$ dans ${ }_{2} \mathrm{OSO}_{4}$, J. Chem. Phys. 88, 1713 (1991).

[34] M. Drillon, M. Belaiche, J. Heintz, G. Vileneuve, A. Boukhari, and J. Aride, Organic and Inorganic Low-Dimensional Crystalline Materials, NATO ASI series, Vol. 168 (Springer Science \& Business Media, 2013), p. 421.

[35] N. Takahashi, S. Okubo, H. Ohta, T. Sakurai, M. Fujisawa, and H. Kikuchi, Dzyaloshinsky-Moriya Interaction Estimated by AFMR of Kagome Like Substance $\mathrm{Cu}_{2} \mathrm{O}\left(\mathrm{SO}_{4}\right)$ Observed at 1.8K, J. Phys.: Conf. Ser. 400, 032097 (2012).

[36] T. Asai, H. Saheki, and R. Kiriyama, Magnetic Studies on Basic Salts of Copper, Dicopper Arsenate Hydroxide, Dicopper Hydroxide Phosphate, and Dicopper Oxide Sulfate, Bull. Chem. Soc. Jpn. 52, 310 (1979).

[37] P. R. Willmott, D. Meister, S. J. Leake, M. Lange, A. Bergamaschi, M. Böge, M. Calvi, C. Cancellieri, N. Casati, A. Cervellino, Q. Chen, C. David, U. Flechsig, F. Gozzo, B. Henrich, S. Jäggi-Spielmann, B. Jakob, I. Kalichava, P. Karvinen, J. Krempasky, A. Lüdeke, R. Lüscher, S. Maag, C. Quitmann, M. L. Reinle-Schmitt, T. Schmidt, B. Schmitt, A. Streun, I. Vartiainen, M. Vitins, X. Wang, and R. Wullschleger, The Materials Science beamline upgrade at the Swiss Light Source, J. Synchrotron Radiat. 20, 667 (2013).

[38] K. Momma and F. Izumi, VESTA3 for three-dimensional visualization of crystal, volumetric and morphology data, J. Appl. Crystallogr. 44, 1272 (2011).

[39] L. Yang, M. Jeong, P. Babkevich, V. M. Katukuri, B. Náfrádi, N. E. Shaik, A. Magrez, H. Berger, J. Schefer, E. Ressouche, 
M. Kriener, I. Živković, O. V. Yazyev, L. Forró, and H. M. Rønnow, $J_{1}-J_{2}$ square lattice antiferromagnetism in the orbitally quenched insulator $\mathrm{MoOPO}_{4}$, Phys. Rev. B 96, 024445 (2017).

[40] H. Papi, V. Y. Favre, H. Ahmadvand, M. Alaei, M. Khondabi, D. Sheptyakov, L. Keller, P. Kameli, I. Živković, and H. M. Rønnow, Magnetic and structural properties of Ni-substituted magnetoelectric $\mathrm{Co}_{4} \mathrm{Nb}_{2} \mathrm{O}_{9}$, Phys. Rev. B 100, 134408 (2019).

[41] P. Bag, P. R. Baral, and R. Nath, Cluster spin-glass behavior and memory effect in $\mathrm{Cr}_{0.5} \mathrm{Fe}_{0.5} \mathrm{Ga}$, Phys. Rev. B 98, 144436 (2018).

[42] S. Pakhira, C. Mazumdar, R. Ranganathan, S. Giri, and M. Avdeev, Large magnetic cooling power involving frustrated antiferromagnetic spin-glass state in $R_{2} \mathrm{NiSi}_{3}(R=\mathrm{Gd}, \mathrm{Er})$, Phys. Rev. B 94, 104414 (2016).

[43] D. W. Rogers, Einstein's “Other” Theory: The Planck-BoseEinstein Theory of Heat Capacity (Princeton University Press, Princeton, NJ, 2005).

[44] J. Rodríguez-Carvajal, Recent advances in magnetic structure determination by neutron powder diffraction, Phys. B (Amsterdam, Neth.) 192, 55 (1993).
[45] C. Ritter, Neutrons Not Entitled to Retire at the Age of 60: More Than Ever Needed to Reveal Magnetic Structures, in Solid Compounds of Transition Elements, Solid State Phenomena Vol. 170 (Trans Tech Publications, Switzerland, 2011), pp. 263-269.

[46] J. Rodriguez-Carvajal, BASIREPS: A program for calculating irreducible representations of space groups and basis functions for axial and polar vector properties, part of the FullProf suite of programs, https://www.ill.eu/sites/fullprof/.

[47] I. Rousochatzakis, S. R. Manmana, A. M. Läuchli, B. Normand, and F. Mila, Dzyaloshinskii-Moriya anisotropy and nonmagnetic impurities in the $s=\frac{1}{2}$ kagome system $\mathrm{ZnCu}_{3}(\mathrm{OH})_{6} \mathrm{Cl}_{2}$, Phys. Rev. B 79, 214415 (2009).

[48] H. M. Rønnow, Structure and magnetic order of the diamondkagome planar compound $\mathrm{Cu}_{2} \mathrm{OSO}_{4}$, STFC ISIS Facility, 2018.

[49] H. M. Rønnow, Magnetic order of the diamond-kagomé planar compound $\mathrm{Cu}_{2} \mathrm{OSO}_{4}$, Institut Laue-Langevin, 2018. 